Asian Journal of Computer Science and Technology

ISSN: 2249-0701 Vol.7 No.2, 2018, pp. 62-65

(C) The Research Publication, www.trp.org.in

\title{
Fruit Disease Detection Using Convolution Neural Network Approach
}

\author{
Shivani and Sharanjit Singh \\ Department of Computer Science, Regional Campus, Guru Nanak Dev University, Gurdaspur, India \\ E-Mail: shivanisharma964223@gmail.com
}

\begin{abstract}
Fruit disease detection is critical at early stage since it will affect the farming industry. Farming industry is critical for the growth of the economic conditions of India. To this end, proposed system uses universal filter for the enhancement of image captured from source. This filter eliminates the noise if any from the image. This filter is not only tackle's salt and pepper noise but also Gaussian noise from the image. Feature extraction operation is applied to extract colour and texture features. Segmented image so obtained is applied with Convolution neural network and $k$ mean clustering for classification. CNN layers are applied to obtain optimised result in terms of classification accuracy. Clustering operation increases the speed with which classification operation is performed. The clusters contain the information about the disease information. Since clusters are formed so entire feature set is not required to be searched. Labelling information is compared against the appropriate clusters only. Results are improved by significant margin proving worth of the study. Keywords: Universal filter, K mean clustering, MSE, CNN
\end{abstract}

\section{INTRODUCTION}

Agriculture plays critical part in harvesting any culture. In fact human civilization greatly depends upon agriculture. Regular Field prevalence and water management in soil is key to achieving goal of good production. Plant and fruit diseases are greatly affecting quality and quantity associated with production. Diseases are common now days. The pesticides and other harmful manure is biggest obstacle in this area. This paper analyzes diseases caused due to fruit harvesting. Image processing techniques are used in order to analyze degradation of fruit crop. Analysis of Filtering techniques associated with distortion detection is presented in comprehensive manner. Also proposed system deals with discovering the problems present within the fruit images. The dataset is extracted from UCI website for proving the worth of the study.

The proposed system uses universal filter to enhance the clarity of the image along with noise handling and performs feature extraction. After extracting features individual colour channel is enhanced in order to perform segmentation. Segmentation is performed using $\mathrm{k}$ means clustering to optimise the result classification is handled using convolution neural network. Convolution neural network mechanism takes the segmented image pixels as population and generates distinct results in terms of classification accuracy. Result is compared against the previous result. This process continues until desired result is obtained.

\section{LITERATURE SURVEY}

Agriculture is critical area for people living. Work has been done toward accomplishing optimality in terms of production and quality. Image processing is the technique used in order to detect diseases present within the image.

Awate \& Deshmankar 2015 [1] proposed mannual monitoring of crops by farmers is extensive task. So techniques have been devised using the applications of artificial neural network to provide automated approach to detect external diseases within the fruit crop.

Anon 2010 proposed disease detection in grapes. This disease is most common among grapes. This disease is devastating in moist environment. This disease affect leafs of grapes which becomes black at borders. The yellowish spot is formed at the centre of leaf which becomes enlarged with time. Preventive mechanism such as fungicides and chemicals are available that decreases the effect of Black rots. [2]

Shah 2016 proposed image processing mechanism in collaboration with machine learning techniques enhances performance of detection. In addition work has been extended to include crop like rice. The detection of problem within rice crop is accomplished using technique of image processing like preprocessing, segmentation, extraction etc. [3]

Ilic et al. 2015 discussed early detection of fruit disease is essential in order to rectify the problems present. Data mining techniques are utilized for this purpose. Image processing techniques requiring optimization also. Automatic approach help enhances finding of problems present within the fruit. Optimization mechanism also help in detection of diseases at early stage hence help in curing process. [4]

Jhuria \& Kum 2013 discussed disease in fruit causes diseases since spread of virus in human body through fruit is exceedingly fast. Apple fruit is commonly infected and cause diseases. Vast amount of research is done in this area. $\mathrm{K}$ means clustering technique is used to filter out the information generated and then distortion is detected. Local binary pattern mechanism available in image processing toolbox is utilized for this purpose. [5]

Engineering et al. 2015 proposed automation as technique which is made to analyze distortion in fruits. In order to 
accomplish this fruit grading system is done. This process of automation utilizes feature extraction. Once features are successfully extracted, classification can be accurately performed. [6]

Sadek 2012 described feature extraction as the process of determining common features and then group or clusters are formulated from which particular values can be extracted to reduce the complexity. In image processing SVD approach is commonly used for this purpose. [7]

Anon n.d. discussed image may become damaged or corrupted on eve of noise. The problem generally occurs due to transmission medium, temperature or any other corruption occurs due to unauthorized access. [8]

Zhang et al. 2015 highlight the algorithm based on low-rank matrix recovery to remove salt \& pepper noise from surveillance video. Unlike single image denoising techniques, noise removal from video sequences aims to utilize both temporal and spatial information. By grouping neighbouring frames based on similarities of the whole images in the temporal domain, we formulate the problem of removing salt \& pepper noise from a video tracking sequence as a low- rank matrix recovery problem. The resulting nuclear norm and L1-norm related minimization problems can be efficiently solved by many recently developed methods. To determine the low-rank matrix, we use an averaging method based on other similar images. [9]

a.M et al. 2014 described the image representation using encoding mechanism. The encoding mechanism which is used is known as Discrete Cosine Transformation. The DCT transformation will use the concept of Fourier Transformation in order to perform encoding and then same process is used in reverse in order to perform decoding. [10]

SStudies 2015 presented the "spherepix" information structure for proficient execution of low-level picture handling operations on circular pictures. Productive execution of lowlevel picture handling depends vigorously on distinguishableness of the convolution parts that frame the key building pieces of generally calculations. Because of the shape of the circle, it is impractical to put an orthogonal framework pixelation all inclusive on its surface, making direct use of established detachable piece convolutions unimaginable. In the spherepix information structure we propose an option approach comprising of a gathering of covering (close orthogonal) matrix patches covering the circle's surface. Near the limits of patches we present information introduction between fix matrices to guarantee data stream between network patches. After each picture handling subroutine, we accommodate information in the covering locales to homogenize the worldwide information portrayal. We guarantee that the extra computational cost of information interjection and information compromise is effectively repaid by the computational sparing and algorithmic effortlessness of applying existing picture preparing subroutines in the framework patches. The approach is exhibited by executing a SIFT include point calculation in spherepix arranges and looking at exactness, review, and computational cost of the proposed way to deal with reported alterations of the SIFT calculation particularly produced for usage on round pictures. [11]

M. Dewdney proposed fungal disease detection mechanism. For observing errors and accuracy Mean root square error mechanism is to be utilized. Accuracy and error rate is inversely proportional to each other.

$$
R M S=\sqrt{\left(x-x_{a}\right)^{2}}
$$

This equation is used to evaluate Root Mean square error. Lower the value of RMS more accurate a prediction. Advantage of this approach is, convergence rate is better but disadvantage is that it can work over limited values. Non negative values are allowed and hence result always lies between 0 and 1. [12]

\section{PROPOSED METHODOLOGY}

Diseases Detection and Classification Procedure:

Input: fruit image

Output: Classified fruit disease

Algorithm:

1. Load a images for training

2. Read image for testing.

3. Perform feature extraction using color features i.e. Global color histogram (GCH), Color coherence vector (CCV) and texture features i.e. Local binary pattern (LBP), complete local binary pattern (CLBP), Local ternary pattern (LTP), Texture Features.

4. Perform feature level fusion using color and texture features.

GCH+TEXTURE
GCH+LTP
TEXTURE+GCH+LBP
TEXTURE+LBP
TEXTURE+CLBP
TEXTURE+LTP
TEXTURE+CLBP+LTP

5. Apply Convolution neural network classifier on the segmented image.

imageInputLayer ([77 1003$])$;

convolution2dLayer(2,6);

ReluLayer(); maxPooling2dLayer(2,'Stride',2);

fullyConnectedLayer(3);

softmaxLayer();

classificationLayer()]

6. If fruit is infected by any disease then go to step 7 otherwise go to step 8 .

7. Apply k mean clustering for image segmentation.

8. Fruit is normal or ordinary fruit.

9. Result the classified fruit disease.

\section{PROPOSED FLOWCHART}

Flowchart describes training and testing image sets required to make accurate decisions regarding predictions. The flow of the system and mechanism used to extract features is given as under: 


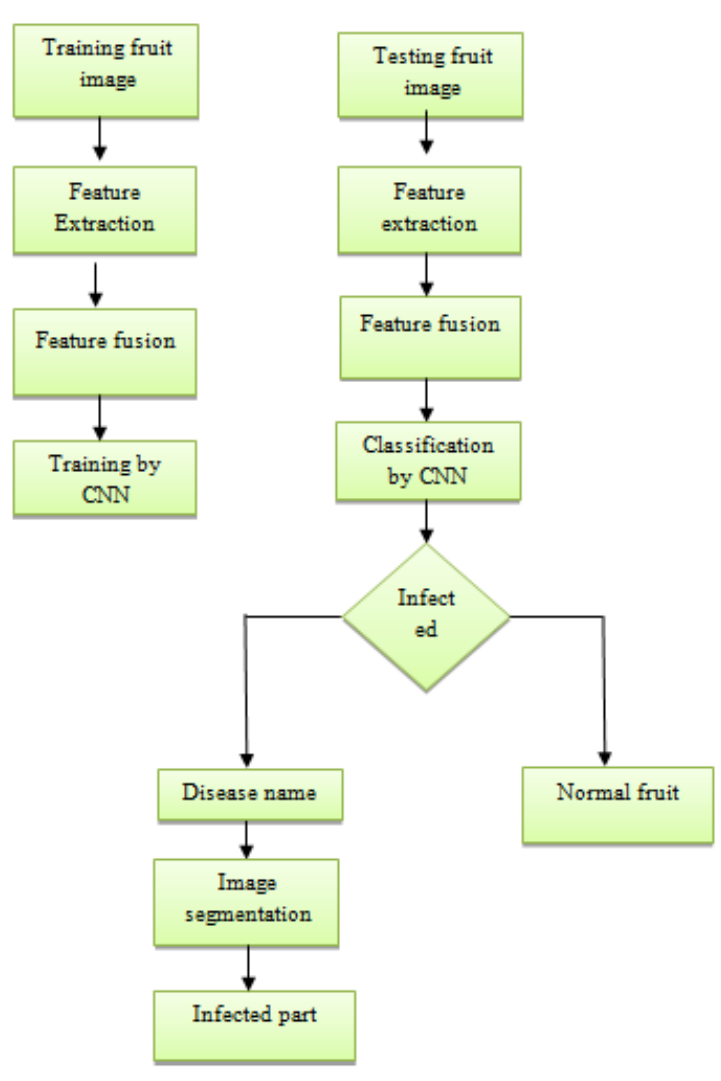

Fig. 1 Flowchart

\section{A. Dataset Description}

Dataset is derived from online source and is of following format

TABLE I IMAGE FORMATS USED IN THE PROPOSED SYSTEM

\begin{tabular}{|c|c|c|c|}
\hline Image Format & Type & Size & Resolution \\
\hline .png & Gray scale & $2 \mathrm{mb}$ & $200 \times 200$ \\
\hline .jpeg & Coloured & $4 \mathrm{mb}$ & $200 \times 200$ \\
\hline .bmp & Black n white & $1 \mathrm{mb}$ & 200 X 200 \\
\hline
\end{tabular}

Dataset derived is given as follows

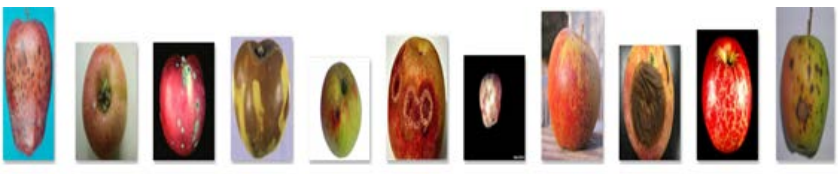

Fig. 2 Images with problems used in the proposed system
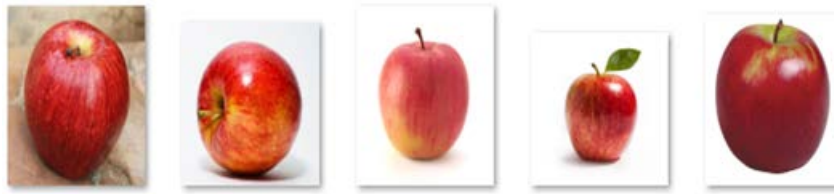

Fig. 3 Normal image dataset used in the proposed system

\section{RESULTS}

Fruit disease detection using universal filter, $\mathrm{k}$ means clustering and convolution neural network algorithm is an efficient technique to detect diseases at early stage and prevent any problems caused to human life. Disease detection suggested through this literature is the method of finding any abnormal form of information images. It is a method of detecting and extracting noisy information and present noise free information for further processing.

In proposed technique noise handling mechanism is introduced to obtain clarity within the image. After obtaining the clarity segmentation and classification is imposed. The image data presented to the simulation is of .jpg and .png type. Results in terms of MSE and PSNR are obtained the desired simulation.

\section{A. Parametric analysis}

Mean square error is obtained by subtracting the features obtained from the proposed system and original image. Lower the MSE lesser is the chances of attack. Signal to noise ratio is another parameter used to evaluate attack. Higher the value of SNR lesser is the chance of attack. Compression reduces the size of the image. Higher the value of compression lesser is the chances of attack. Result in terms of tables with various parameters is given as under:

TABLE II MSE COMPARISON

\begin{tabular}{|c|c|c|}
\hline Image set & MSE Existing & MSE Proposed \\
\hline Image1 & 14.0869 & 7.04345 \\
\hline Image2 & 15.7442 & 7.87209 \\
\hline Image3 & 132.03 & 66.015 \\
\hline Image4 & 14.0869 & 7.04345 \\
\hline Image5 & 31.7646 & 15.8823 \\
\hline Image6 & 1.38107 & $1.00486 \mathrm{e}-012$ \\
\hline Image7 & 26.5165 & 13.2583 \\
\hline Image8 & $2.00972 \mathrm{e}-012$ & $1.00486 \mathrm{e}-012$ \\
\hline Image9 & 45.5752 & 22.7976 \\
\hline
\end{tabular}

Plots for MSE are given as under:

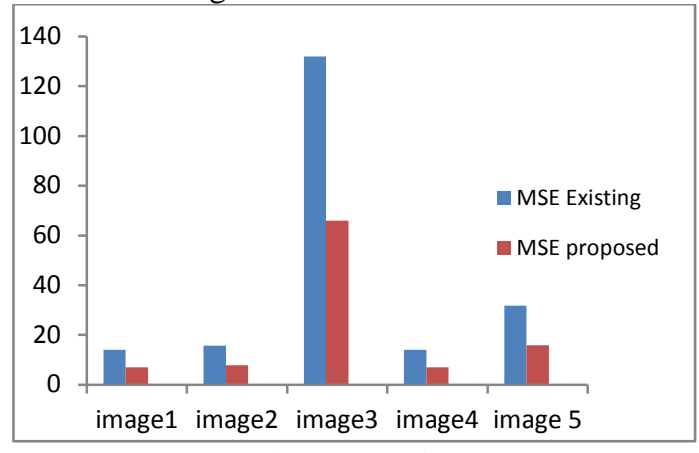

Fig. 4 Graph of MSE 


\section{CONCLUSION AND FUTURE SCOPE}

The proposed system uses universal filter at pre-processing stage. Pre-processing stage is crucial and is used to enhance the image along with its clarity. The clarity of the image determines the final result accuracy. Next phase is feature extraction. Features are extracted in the form of textured and colored features. Segmentation through the k-means clustering is performed. The primary objective of this approach is to give critical and non-critical image segment division. Classification through convolution neural network is obtained. Convolution neural network is yield optimal result in terms of classification accuracy.

In future, real time datasets can be tested against the proposed system. Also convergence rate can be further improved using prompted random forest mechanism.

\section{REFERENCES}

[1] Awate and D. Deshmankar, "Fruit Disease Detection using Color, Texture Analysis and ANN”, pp. 970-975, 2015.

[2] "Survey on Major Diseases of Vegetable and Fruit Crops Total Number of leaves / Fru it counted Sum of all disease ratings $\times 100$
Total number of leaves / fru its $\times$ maximum rating value”, Vol. 35 , No. September, pp. 423-429, 2010.

[3] J. P. Shah, "A Survey on Detection and Classification of Rice Plant Diseases”, 2016.

[4] M. Ilic, P. Spalevic, M. Veinovic, and A. A. M. Ennaas, "Data mining model for early fruit diseases detection”, pp. 910-913, 2015.

[5] M. Jhuria and A. Kum, "g for Smart Farming: Detection of Dise ase and Fruit Grading”, pp. 521-526, 2013.

[6] Engineering, A. Kumar, and G. S. Gill, "Automatic Fruit Grading and Classification System Using Computer Vision : A Review”, No. I, pp. 598-603, 2015.

[7] R. A. Sadek, "SVD Based Image Processing Applications: State of The Art, Contributions and Research Challenges”, Vol. 3, No. 7, pp. 26-34, 2012.

[8] Y. Zhang, Y. Liu, X. Li, and C. Zhang, "Salt and pepper noise removal in surveillance video based on low-rank matrix recovery," Comput. Vis. Media, Vol. 1, No. 1, pp. 59-68, Aug. 2015.

[9] R. a.M, K. W.M, E. M. a, and W. Ahmed, "Jpeg Image Compression Using Discrete Cosine Transform - A Survey”, Int. J. Comput. Sci. Eng. Surv., Vol. 5, No. 2, pp. 39-47, 2014.

[10] Science and M. Studies, "Use of Digital Image Processing for Grain Counting”, pp. 6-9, 2015.

[11] M. Dewdney, "Fungal Diseases of Citrus Fruit and Foliage Foliar Fungal Diseases to be Covered", IEEE 17th Int. Conf. Parallel Distrib. Syst., 2014. 